E791 Physics Workshop

University of California

Santa Cruz, California USA

27-31 July 1992

\title{
CHARM STRANGE BARYONS AND THE 1.5 PRONG
}

\author{
D. J. Summers \\ Dept. of Physics and Astronomy \\ University of Mississippi \\ Oxford, Mississippi 38677 USA
}

CONF-9207178--2

DE93 006022

The charm strange baryons, $\Xi_{c}^{0}, \Xi_{c}^{+}$, and $\Omega_{c}^{0}$, are most reliably tagged as baryons by their decay into charged hyperons. Six of the nine decay modes of these charm strange baryons, which have currently been observed (Table 1), exhibit such decays. The charged hyperon travels about a meter with wide variations (Table 4). This can give rise to a track which is seen in the silicon microstrip detector (SMD) but not further downstream. To use the charged hyperon to form a vertex, the silicon microstrip stub must be found. In two out of four of the observed decays of the $\Xi_{c}^{0}$ the stub must be combined together with only one other track to form a vertex; hence the title 1.5 prong. A real 2-prong is not available for a topological vertexer to find. A topological vertexer also misses $\Xi_{c}^{+} \rightarrow \Xi^{-} \pi^{+} \pi^{+}$because it appears to be a 2 -prong with charge $=+2$. The short lifetimes of charm strange baryons again hamper a plain topological vertex filter.

But all is not lost. Many of the hyperons, which are relatively rare in normal events but common in charm strange baryon events, decay into a $\Lambda$. This and perhaps other tags can be used to supplement a vertex filter. These $\Lambda$ s will appear to have an unusually long lifetime, because they have originated from a hyperon decay downstream of the target. This is a further tag that they have indeed come from a hyperon decay. It may also be possible to find and filter hyperons which do not decay into $\Lambda \mathrm{s}$ with adequate efficiency to determine charm strange baryon branching ratios involving them. That is the good news. The bad news is that the $\Lambda$ s often der $y$ in the first E791 analyzing magnet and techniques must be optimized to find and filter on these long-lived $\Lambda$ s. Those that decay downstream of D2 cannot be completely momentum analyzed. A few charged hyperons will make it into D2 allowing direct momentum measurement; but in these cases not much decay space is left for a $\Lambda$.

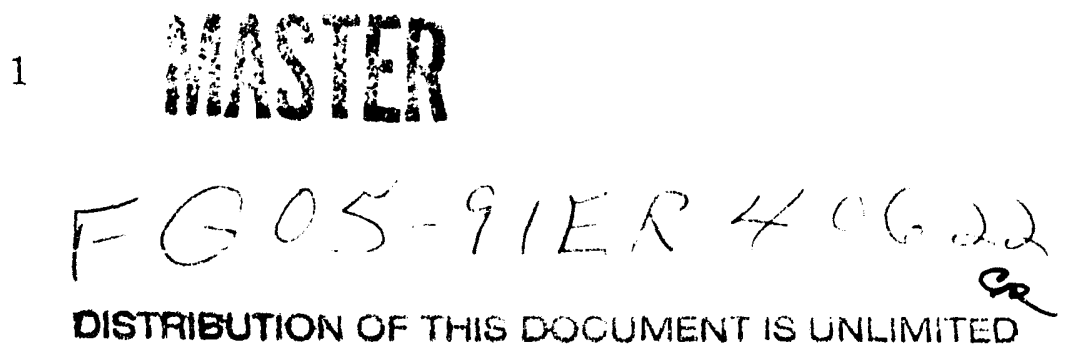


In summary, new filtering techniques must be developed in addition to a topological vertex filter to adequately exploit charm strange baryons. This work was supported by the U.S. Department of Energy (DE-FG05-91ER40622).

Table 1. Charm Strange Baryon Decay Modes

Observed

$$
\begin{aligned}
& \Xi_{c}^{0} \rightarrow \Xi^{-} \pi^{+} \\
& \Xi_{c}^{0} \rightarrow \Xi^{-} \pi^{+} \pi^{+} \pi^{-} \\
& \Xi_{c}^{0} \rightarrow p K^{-} K^{*}(892)^{0} \\
& \Xi_{c}^{0} \rightarrow \Omega^{-} K^{+} \\
& \Xi_{c}^{+} \rightarrow \Lambda K^{-} \pi^{+} \pi^{+} \\
& \Xi_{c}^{+} \rightarrow \Sigma^{+} K^{-} \pi^{+} \\
& \Xi_{c}^{+} \rightarrow \Sigma^{0} K^{-} \pi^{+} \pi^{+} \\
& \Xi_{c}^{+} \rightarrow \Xi^{-} \pi^{+} \pi^{+} \\
& \Omega_{c}^{0} \rightarrow \Xi^{-} K^{-} \pi^{+} \pi^{+}
\end{aligned}
$$

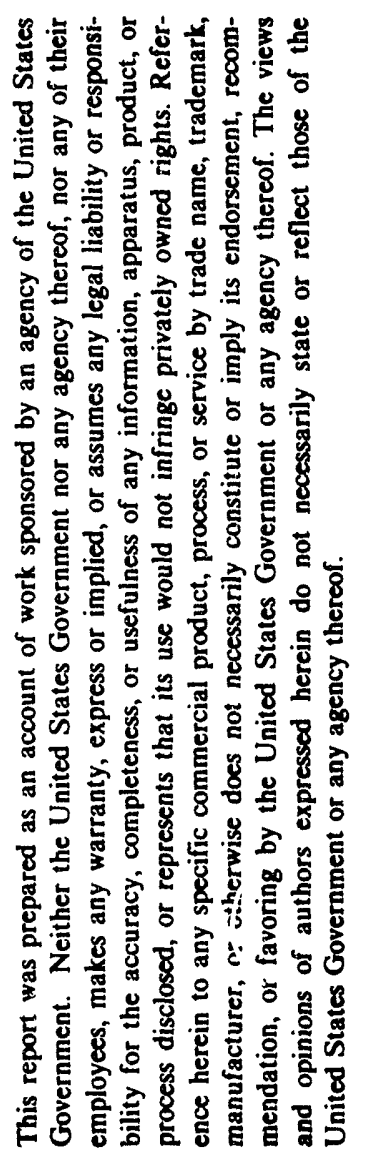

Table 2. Branching Ratios of Decay Products

$\begin{array}{llr}\text { Particle } & \text { Decay Mode } & \% \\ K_{\mathrm{S}}^{0} & \pi^{+} \pi^{-} & 68.6 \\ \bar{K}^{*}(892)^{0} & K^{-} \pi^{+} & 67 \\ \phi & K^{+} K^{-} & 49.1 \\ \Lambda & p \pi^{-} & 64.1 \\ \Sigma^{-} & n \pi^{--} & 99.8 \\ \Sigma^{0} & \Lambda \gamma & 100 \\ \Sigma^{+} & p \pi^{0} & 51.6 \\ & n \pi^{+} & 48.3 \\ \Xi^{-} & \Lambda \pi^{-} & 100 \\ \Xi^{0} & \Lambda \pi^{0} & 100 \\ \Omega^{-} & \Lambda K^{-} & 67.8 \\ & \Xi^{0} \pi^{-} & 23.6\end{array}$

Unobserved (Partial List)

$$
\begin{aligned}
& \Xi_{c}^{0} \rightarrow \Lambda K_{\mathrm{S}}^{0} \\
& \Xi_{c}^{0} \rightarrow \Xi^{-} e^{+} \nu_{e} \\
& \Xi_{c}^{0} \rightarrow \Xi^{-} \mu^{+} \nu_{\mu} \\
& \Xi_{c}^{0} \rightarrow \Sigma^{-} K^{-} \pi^{+} \pi^{+} \\
& \Xi_{c}^{0} \rightarrow \Xi^{0} \phi
\end{aligned}
$$

$\Xi_{+}^{c} \rightarrow \Lambda K_{\mathrm{S}}^{0} \pi^{+}$

$\Xi_{c}^{+} \rightarrow \Xi^{-} \pi^{+} e^{+} \nu_{e}$

$\Xi_{c}^{+} \rightarrow \Xi^{-} \pi^{+} \mu^{+} \nu_{\mu}$

$\Omega_{c}^{0} \rightarrow \Omega^{-} \pi^{+}$

$\Omega_{c}^{0} \rightarrow \Omega^{-} e^{+} \nu_{e}$

$\Omega_{c}^{0} \rightarrow \Omega^{-} \mu^{+} \nu_{\mu}$ 
Table 3. E791 Spectrometer Z Locations

$\begin{array}{lc} & \text { Z }(\mathrm{cm}) \\ \text { First Target } & -8.2 \\ \text { Last Target } & -2.1 \\ \text { First SMD } & 0.7 \\ \text { Last SMD } & 45.5 \\ \text { PWC \#9 } & 118.5 \\ \text { First D1 Plane } & 142.5 \\ \text { Last D1 Plane } & 183.6 \\ \text { Middle of Magnet 1 } & 272.2 \\ \text { First D2 Plane } & 382.4 \\ \text { Last D2 Plane } & 500.9\end{array}$

Table 4. Decay Lengths of Weakly Decaying Paricicles for 3 Momenta

\begin{tabular}{|c|c|c|c|c|c|c|}
\hline Particle & Quarks & $\begin{array}{l}\text { Mass } \\
\left(\mathrm{MeV} / c^{2}\right)\end{array}$ & $\begin{array}{r}c \tau \\
(\mathrm{cm})\end{array}$ & $\begin{array}{r}20 \mathrm{Gev} / \mathrm{c} \\
(\mathrm{cm})\end{array}$ & $\begin{array}{r}50 \mathrm{GeV} / \mathrm{c} \\
(\mathrm{cm})\end{array}$ & $\begin{array}{r}100 \mathrm{Gev} / \mathrm{c} \\
(\mathrm{cm})\end{array}$ \\
\hline$\Omega_{c}^{0}$ & ssc & 2719 & & & & \\
\hline$\Xi_{c}^{0}$ & dsc & 2472.7 & .0025 & .02 & .05 & .10 \\
\hline$\Xi_{c}^{+}$ & usc & 2466.4 & .0090 & .07 & .18 & .36 \\
\hline$\Lambda_{c}^{+}$ & udc & 2284.9 & .0057 & .05 & .12 & .25 \\
\hline$D^{0}$ & $c \bar{u}$ & 1864.5 & .0126 & .14 & .34 & .68 \\
\hline$D_{\mathrm{s}}^{+}$ & $\mathrm{c} \overline{\mathbf{s}}$ & 1968.8 & .0135 & .14 & .34 & .69 \\
\hline$D^{+}$ & $c \bar{d}$ & 1869.3 & .0320 & .34 & .86 & 1.71 \\
\hline $\bar{B}^{0}$ & $\mathrm{~b} \overline{\mathrm{d}}$ & 5278.7 & .0387 & .15 & .37 & .73 \\
\hline$B^{-}$ & $\mathrm{b} \overline{\mathbf{u}}$ & 5278.6 & .0387 & .15 & .37 & .73 \\
\hline $\bar{K}^{0}$ & $s \bar{d}$ & 497.7 & $K_{\mathrm{S}}^{0} \quad 2.68$ & 108 & 269 & 538 \\
\hline$\Lambda$ & uds & 1115.6 & 7.89 & 141 & 354 & 707 \\
\hline$\Sigma^{-}$ & $\mathrm{dds}$ & 1197.4 & 4.43 & 74 & 185 & 370 \\
\hline$\Sigma^{+}$ & uus & 1189.4 & 2.39 & 40 & 100 & 201 \\
\hline$\Xi^{-}$ & dss & 1321.3 & 4.91 & 74 & 186 & 372 \\
\hline$\Xi^{0}$ & uss & 1314.9 & 8.69 & 132 & 330 & 661 \\
\hline$\Omega^{-}$ & sss & 1672.4 & 2.46 & 29 & 74 & 147 \\
\hline
\end{tabular}




\section{BIBLIOGRAPHY}

[1] H. Albrecht et al. (ARGUS), Evidence for the Production of the Charmed, Doubly Strange Baryon $\Omega_{c}$ in $e^{+} e^{-}$Annihilation, Physics Letters $\underline{B 288}$ (1992) 367; DES:-92-052.

[2] D. MacFarlane et al. (ARGUS), Selected Charm and Tau Results from ARGUS, XXVIIth Rencontre de Moriond, Electroweak Interactions and Unified Theories, Les Arcs, France (15-22 March 1992).

[3] H. Albrecht et al. (ARGUS), Measurement of $\Xi_{c}$ Production in $e^{+} e^{-}$Annihilation at $10.5 \mathrm{GeV}$ Center-of-Mass Energy, Physics Letters B247 (1990) 121.

[4] S. Henderson et al. (CLEO), Observation of the Decay $\Xi_{c}^{0} \rightarrow \Omega^{-} K^{+}$, Physics Letters $\underline{B 283}$ (1992) 161; CLNS-91-1125.

[5] P. Avery et al. (CLEO), Observation of the Charmed Strange Baryon $\Xi_{c}^{0}$, Phys. Rev. Lett. $\underline{62}$ (1989) 863.

[6] M.S. Alam et al. (CLEO), Measurement of the Isospin Mass Splitting $\Xi_{c}^{+}-\Xi_{c}^{0}$, Physics Letters B226 (1989) 401.

[7] S. Barlag et al. (ACCMOR), First Measurement of the Lifetime of the Charmed Strange Baryon $\Xi_{c}^{0}$, Physics Letters $\underline{B 236}$ (1990) 495.

[8] S. Barlag et al. (ACCMOR), Measurement of the Mass and Lifetime of the Charmed Strange Baryon $\Xi_{c}^{+}$, Physics Letters $\underline{B 233}$ (1989) 522.

[9] S.F. Biagi et al. (WA62), Properties of the Charmed Strange Baryon $\mathrm{A}^{+}$and Evidence for the Charmed Doubly Strange Baryon $T^{0}$ at $2.74-\mathrm{GeV} / c^{2}$, Zeitschrift für Physik $\underline{C 28}$ (1985) 175.

[10] S.F. Biagi et al., Measurement of the Lifetime of the Charmed Strange Baryon $A^{+}$, Physics Letters $\underline{150 B}(1985) 230$.

[11] S.F. Biagi et al., Observation of a Narrow State at $2.46 \mathrm{GeV} / c^{2}-\mathrm{A}$ Candidate for the Charmed Strange Baryon $A^{+}$, Physics Letters $\underline{122 B}$ (1983) 455.

[12] P. Coteus et al. (E400), Production of the Charmed Strange Baryon $\Xi_{c}^{+}$by Neutrons, Phys. Rev. Lett. $\underline{59}$ (1987) 1530.

[13] C. Itoh, T. Minamikawa, K. Miura, and T. Watanabe, $\Xi_{c}$ and Baryon Spectroscopy, Phys. Rev. D40 (1989) 3660.

[14] S. Fleck and J.M. Richard, Charmed Baryons, Particle World 1 (1990) 67.

[15] J.G. Korner and H.W. Siebert, Charm Baryons: Theory and Experiment, Annual Review of Nuclear and Particle Physics $\underline{41}$ (1991) 511-545. 

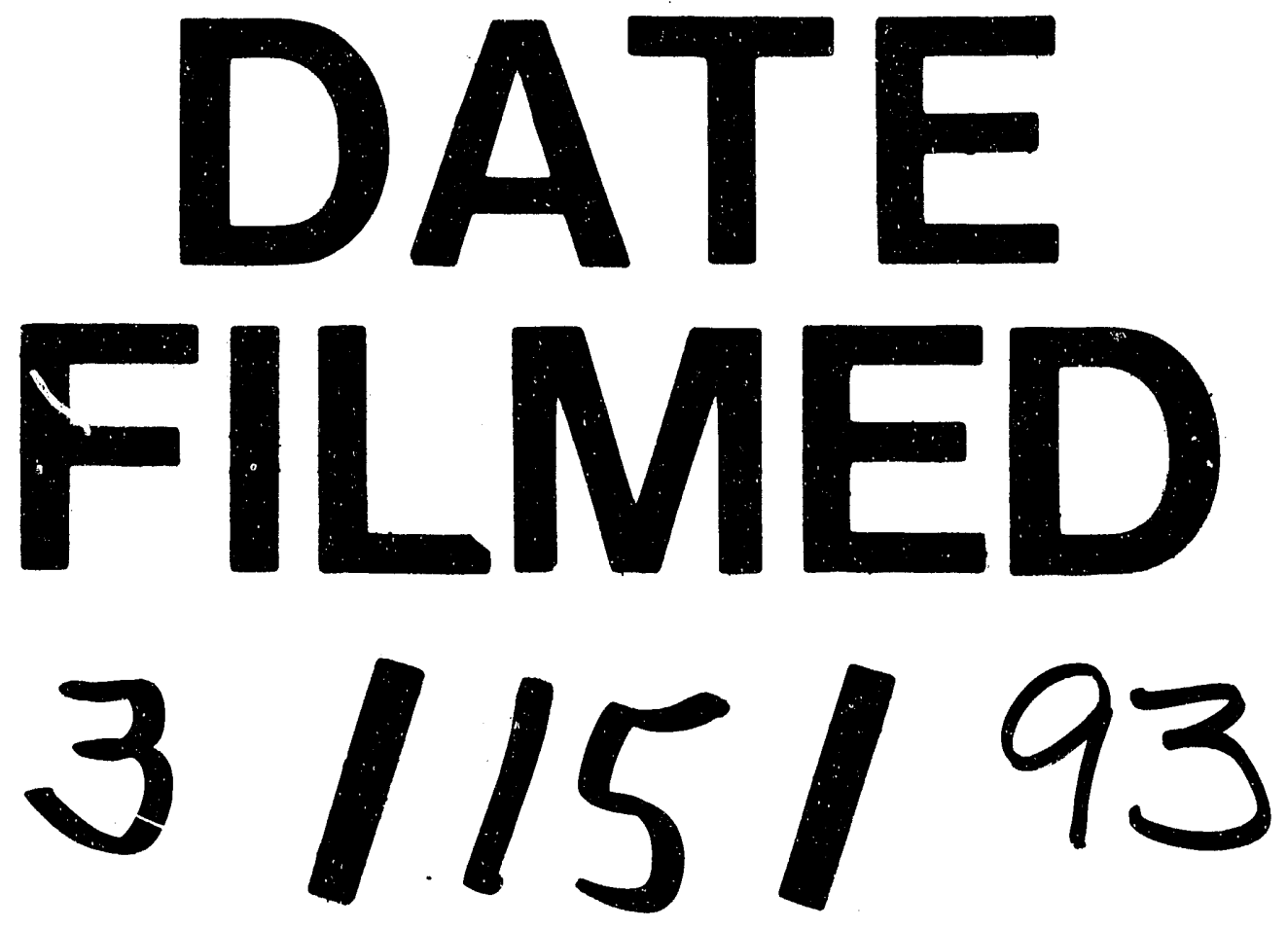
